\title{
A Review on Thermodynamic Optimization of Irreversible Refrigerator and Verification with Transcritical $\mathrm{CO}_{2}$ System
}

\author{
Jahar Sarkar* \\ Department of Mechanical Engineering \\ IIT (BHU) Varanasi, India \\ E-mail: js_iitkgp@yahoo.co.in
}

\begin{abstract}
The transcritical vapor compression refrigeration cycle consists of isothermal heat addition process and isobaric non-isothermal heat rejection process with highly variable heat capacity unlike to the subcritical cycle. Hence, it is quite interesting whether the analysis and optimization results of irreversible Carnot-like refrigerator are applicable for this case. The present study consists of two parts: the detailed review on theoretical analysis and analytical optimizations of irreversible Carnot-like refrigerator with both infinite and finite capacity heat reservoirs, and verification of analytical results with the results obtained from a more elaborate numerical simulation of transcritical $\mathrm{CO}_{2}$ refrigeration system. Considered objective parameters are cooling load or COP and overall heat transfer surface area, and optimizing parameters are cold and hot side working fluid temperatures, ratio of residence times for heat addition and heat rejection, and heat transfer surface area ratio. Reasonably fair agreement has been obtained between analytical and numerical model predictions.
\end{abstract}

Keywords: Irreversible refrigerator; Finite time thermodynamics; Cooling load; COP; Heat exchanger area; Transcritical $\mathrm{CO}_{2}$ system; Numerical verification.

\section{Introduction}

The Carnot (reversible) refrigeration cycle is generally known as being the best cycle operating between two heat reservoirs. However, this type of cycle is practically not feasible, because a reversible process must be carried out at an infinitesimally slow pace and a finite amount of heat transferred in a reversible process requires an infinitesimally small temperature difference and hence a finite amount of cooling load provided by the reversible refrigerator needs an infinitely large heat exchanger. On the other hand, isentropic compression and expansion are not realistic. Therefore, the real vapor compression refrigeration cycle experiences two types of irrevesibilities: (i) internal irreversibility mainly associated with compression and expansion, (ii) external irreversibility mainly associated with heat addition and heat rejection with reservoirs. Based on irreversibility considered, the Carnotlike refrigeration cycle can be classified as four categories: (i) reversible (both internally and externally reversible), (ii) exoreversible (internally irreversible but externally reversible), (iii) endoreversible (internally reversible but externally irreversible and (iv) irreversible (both internally and externally irreversible), which can be named as irreversible Carnot-like cycle [1]. One of the topics in thermodynamics, so-called finite time thermodynamics (FTT) or irreversible thermodynamics, has been the formulation of criteria for comparing the performances between real and ideal cycles. The finite-time thermodynamics basically simplify real cycles by using some specific parameters to avoid the complexity of real processes (real fluid properties and heat transfer and fluid flow effects in real components). A major objective of finite-time thermodynamics is to understand irreversible, finite-time processes and to establish the general, natural bounds on the COP and maximum cooling load or heating load and to establish general operation principles for systems which serve as models for real processes [2-5]. Leff and Teeters [6] first noted that there is a close relationship between the energy efficiency ratio of an air conditioner unit and the coefficient of performance (COP) of its refrigeration cycle, which helps to bridge the gap between pure thermodynamics and practical energy related problems. Keeping this in mind, Blanchard [7] gave the COP for a finite-speed heat pump operated at minimum input power for given heating load. Bejan [8] first introduced the internal heat leak between reservoirs in the thermodynamic model. After the seminal studies by Leff and Teeters, Blanchard and Bejan, a large number of studies have been reported on thermodynamic and finite time optimization of refrigeration and heat pump cycles [950]. Work done on this field considered the followings: (i) endoreversible or irreversible cycle, (ii) with or without heat leak, (iii) linear heat transfer ( $\propto \Delta T$ ) or nonlinear heat transfer $\left(\propto \Delta\left(T^{n}\right)\right.$ and $\left.\propto(\Delta T)^{n}\right)$, single or combined heat transfer mode and (iv) infinite or finite reservoir.

Numerical verification or experimental validation of the analytical results is valuable to the design engineer. Although works related to verification with actual refrigeration cycle are limited. Chen et al. [34] optimized the area ratio and confirmed it against numerical simulation of actual refrigeration and air conditioning plants. Petre et al. [39] used experimental data for comparison with analytical one. Sarkar and Bhattacharyya [37] and Sarkar et al. [36] verified their analytical results with the more elaborate numerical optimization results obtained for ammonia based vapour compression refrigeration and heat pump systems working with both infinite and finite capacity reservoirs. All these verifications were done with 
subcritical cycle, which is very near to irreversible Carnotlike cycle. However, the transcritical $\mathrm{CO}_{2}$ refrigeration cycle doesn't consist of isothermal heat rejection process. $\mathrm{CO}_{2}$ is a promising refrigerant now-a-days as the conventional synthetic working fluids are being phased out worldwide to combat with the twin menace of ozone layer depletion and global warming [51-52]. It is now quite interesting whether the irreversible Carnot-like cycle model is applicable for transcritical cycle.

In the present study, energetic optimization results of irreversible Carnot-like refrigeration cycle with linear heat transfer model for both constant and variable temperature heat reservoirs have been reviewed. Analytical results have been verified with the transcritical $\mathrm{CO}_{2}$ refrigeration cycle as well.

\section{Analytical Optimization with Infinite Heat Reservoirs 2.1 Problem Formulation}

The model of an irreversible refrigeration cycle with constant temperature (infinite heat capacity) reservoirs, as shown in Figure 1, incorporates two types of irreversibilities: i) irreversibility due to heat transfer between two fluids and ii) irreversibilities associated with compression and expansion processes. For finite heat transfer to occur between the reservoir and the working fluid in both low temperature and high temperature sides, a finite temperature difference is required for a finite size heat exchanger. So, when the two isothermal processes occur, the working fluid temperatures are different from the reservoir temperatures and there exist a relation $T_{W}>T_{H}>T_{L}>T_{C}$. The present review summarizes the analytical works on irreversible refrigeration cycle, which were done based on the following assumptions:

(i) The heat capacities of the heat source and heat sink are infinite, so that heat source and heat sink temperatures remain constant in the heat transfer processes.

(ii) The overall heat transfer coefficients $\left(U_{L}\right.$ and $\left.U_{H}\right)$ in the heat exchangers between the refrigerator and its surrounding heat reservoirs are constant.

(iii) The heat transfers are continuous and steady.

(iv) The internal irreversibility (mainly associated with compression and expansion) is considered by using a single parameter (irreversibility parameter).

(v) External irreversibilities are caused by heat-transfers in the high- and low- temperature side heatexchangers between the refrigerator and its surrounding heat reservoirs.

(vi) Linear heat transfer model which is very common in practical applications.

(vii) Compression and expansion are so quick that the time requirements are negligible relative to heat addition and heat rejection.

Following second law of thermodynamics can be applied to the working fluid, undergoing an irreversible Carnot-like refrigerator [14]:

$$
\frac{Q_{H}}{Q_{L}}=\frac{T_{W}\left(S_{2}-S_{3}\right)}{T_{C}\left(S_{1}-S_{4}\right)} \quad \text { or, } \quad \frac{Q_{H}}{Q_{L}}=\phi \frac{T_{W}}{T_{C}}
$$

where, the irreversibility parameter $\phi$ is the ratio of entropy loss and entropy gain by working fluid with hot and cold reservoir respectively ( $\phi \geq 1)$. This parameter takes care of the cycle internal irreversibility occurring due to friction, turbulence, nonequilibrium, etc. The generic source of internal irreversibility is measured by this single irreversibility factor which transforms the Clausius inequality into an equality to simplify the cycle model. It has been taken as constant for derivation. However, it is dependent on both hot and cold fluids temperatures, compression efficiency and fluid properties in real system. Hence, the optimization results will be more reliable if real data based proper relationship of it with $T_{W}$ and $T_{C}$ is introduced.

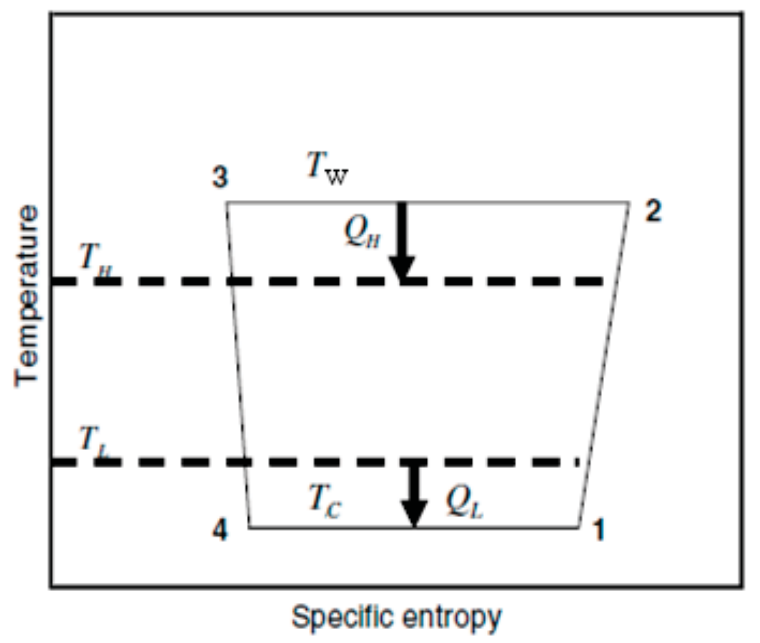

Figure 1. Irreversible refrigerator with infinite heat reservoirs.

\subsection{Optimization Results}

Maximization of COP or cooling capacity is the most primary criteria for designing a refrigerator, which was done by many authors started with Blanchard [7]. Using FTT, heat transfers are given by,

$Q_{L}=U_{L} A_{L}\left(T_{L}-T_{C}\right) t_{C}$

$Q_{H}=U_{H} A_{H}\left(T_{W}-T_{H}\right) t_{W}$

Using Eqs. (1-3) and based on the stated assumptions, the relation of the cooling load is given by [35]:

$\dot{Q}_{L}=\frac{Q_{L}}{t_{W}+t_{C}}=\left[\frac{\phi\left(T_{W} / T_{C}\right)}{U_{H} A_{H}\left(T_{W}-T_{H}\right)}+\frac{1}{U_{L} A_{L}\left(T_{L}-T_{C}\right)}\right]^{-1}$

Using Eq. (1), the coefficient of performance (COP) is given by:

$C O P=\frac{Q_{L}}{Q_{H}-Q_{L}}=\frac{T_{C}}{\phi T_{W}-T_{C}}$

$\Rightarrow \frac{T_{C}}{T_{W}}=\phi \frac{C O P}{C O P+1}=\phi X$

Unlike the finite-time maximum power cycle, the irrreversible refrigeration cycle can be optimized with respect to only one free variable, i.e. refrigeration capacity should be specified to maximize COP or the COP should be specified to maximize cooling capacity, when reservoirs are the constraints. Using Eqs. (4) and (5), the working fluid temperatures can be optimized to get maximum cooling load for given COP and the correlations for the optimum cold and hot working fluid temperatures and maximum cooling load are given by: 


$$
\begin{aligned}
& T_{C, \text { opt }}=\left(\phi X \sqrt{\alpha \delta} T_{H}+\sqrt{\phi} T_{L}\right) /(\sqrt{\alpha \delta}+\sqrt{\phi}) \\
& T_{W, \text { opt }}=\left(\sqrt{\alpha \delta} T_{H}+\phi^{-1 / 2} X^{-1} T_{L}\right) /(\sqrt{\alpha \delta}+\sqrt{\phi}) \\
& \dot{Q}_{L, \text { max }}=\left[U_{H} A_{H}(\sqrt{\alpha \delta}+\sqrt{\phi})^{-2}\right]\left(T_{L}-\phi X T_{H}\right)
\end{aligned}
$$

where, ratios of heat transfer coefficients and areas are given by, $\alpha=A_{H} / A_{L}$ and $\delta=U_{H} / U_{L}$. Chen [14] maximized COP for given cooling load by considering the heat leaks (which is not so important for vapor compression refrigeration cycle) also and found similar correlations for optimum working fluid temperatures. Bhardwaj et al. [53] found similar results for given heating load by minimizing work input to the heat pump and showed that internal irreversibility is more responsible compared to external irreversibility for performance reduction. Assad [54] maximized cooling load with combined convection and radiation heat transfer. However, it is mainly dependent on actual component design. Sarkar et al. [35] have maximized the combined cooling and heating capacity and obtained similar expressions. They have also optimized the residence time ratio and got following expression for optimum time allocation:

$$
\left(t_{W} / t_{C}\right)_{o p t}=\sqrt{\phi} / \sqrt{\alpha \delta}
$$

Again, the residence time in real heat exchangers is dependent on flow condition and configuration. Another important optimizing parameter is area allocation to get maximum cooling load or COP, which was started with the work by Bejan [12]. Heat transfers in rate form are given by [25],

$$
\begin{aligned}
& \dot{Q}_{L}=U_{L} A_{L}\left(T_{L}-T_{C}\right) \\
& \dot{Q}_{H}=U_{H} A_{H}\left(T_{W}-T_{H}\right)
\end{aligned}
$$

Using Clausius inequality in rate form [25],

$$
\dot{Q}_{H} / \dot{Q}_{H}=\phi T_{W} / T_{C}
$$

Using Eqs. (10-12), for given load, the COP can also be expressed by,

$$
C O P=\left\{\phi T_{H} /\left[T_{L}-\dot{Q}_{L}(1+\alpha)\left(\phi / U_{H}+\alpha / U_{L}\right) /\left(\alpha A_{t}\right)\right]-1\right\}^{-1}
$$

where, total heat transfer surface area of the two heat exchangers, $A_{t}=A_{L}+A_{H}$.

Based on stated assumptions and considering total area as constant, the optimum heat transfer surface area ratio for maximum COP is given by [25],

$$
\alpha_{\text {opt }}=\sqrt{\phi / \delta}=\left(\phi U_{L} / U_{H}\right)^{1 / 2}
$$

Hence, the maximum COP will be given by [25],

$$
C O P_{\max }=\left[T_{L}-B \dot{Q}_{L}\right] /\left[\left(\phi T_{H}-T_{L}\right)+B \dot{Q}_{L}\right]
$$

Where, $B=(1+\sqrt{\delta / \phi})^{2} /\left(U_{H} A_{t}\right)$.

El-Din [31] maximized the cooling load, for given COP, by analyzing the totally irreversible refrigerator for total heat transfer area constraint and found similar expression for an optimum balance between the sizes of the heat exchangers at the hot and cold ends of the machine. The following correlations were derived for heat transfer surface area ratio distribution for cold and hot ends:

$$
\begin{aligned}
& A_{L, o p t}=A_{t} /(1+\sqrt{\phi / \delta}) \\
& A_{H, o p t}=A_{t} \sqrt{\phi / \delta} /(1+\sqrt{\phi / \delta})
\end{aligned}
$$

The maximum rate of heat removed from the refrigerated space corresponding to the optimum heat transfer area ratio is given by,

$$
\dot{Q}_{L, \max }=\frac{U_{H} A_{t} T_{H}\left[\left(T_{L} / T_{H}\right)-\phi X\right]}{(\sqrt{\delta}+\sqrt{\phi})^{2}}
$$

Chen et al. [34] maximized the COP of irreversible refrigerator by considering heat leaks also for given cooling capacity to get optimum allocation of heat transfer areas and found similar expressions for optimum area ratio and corresponding optimum heat transfer surface area allocation. Hence, the expressions for optimum area distribution are valid for both maximum COP and cooling capacity for given alternate as both represent same [35]. Chen et al. [34] showed the trend of various parameters calculated based on simulation using data of actual airconditioning plant, although the comparison with their analytical works was not done. Qureshi and Zubair [55] optimized cost of heat exchanger inventory for mechanical subcooling refrigeration cycles.

Minimization of heat exchanger area for certain capacity is very important in the design of refrigeration and heat pump systems yielding space, weight and cost benefits. Sarkar et al. [36] analytically minimized the total area per unit capacity of refrigeration systems for constant temperature heat sources and sinks considering both internal and external irreversibilities. Expressions were obtained for optimum refrigerant temperatures and heat exchanger area ratios. Using Eqs. (10-12), the total heat exchanger area required for unit cooling output is given by,

$\frac{A_{L}}{\dot{Q}_{L}}=\frac{\phi T_{W} / T_{C}}{U_{H}\left(T_{W}-T_{H}\right)}+\frac{1}{U_{L}\left(T_{L}-T_{C}\right)}$

Based on stated assumptions, the following optimal working fluid temperatures and minimum total heat transfer area were obtained:

$$
\begin{aligned}
& T_{W, \text { opt }}=\left[\sqrt{\delta} T_{H}+T_{L} /(X \sqrt{\phi})\right] /(\sqrt{\phi}+\sqrt{\delta}) \\
& T_{C, \text { opt }}=\left(\phi \sqrt{\delta} X T_{H}+T_{L} \sqrt{\phi}\right) /(\sqrt{\phi}+\sqrt{\delta})
\end{aligned}
$$

$\left(A_{t} / \dot{Q}_{L}\right)_{\min }=(\sqrt{\phi}+\sqrt{\delta})^{2} /\left[U_{H}\left(T_{L}-\phi X T_{H}\right)\right]$ 
The variation of $\phi$ was taken as constant probably due to negligible variation with fluid temperatures. The optimal cooling COP corresponding to the minimum overall heat transfer area can be written as:

$C O P_{\text {opt }}=T_{L}^{*} /\left[\phi T_{H}-T_{L}^{*}\right]$

where, $T_{L}^{*}=T_{L}-(\sqrt{\phi}+\sqrt{\delta})^{2} /\left[U_{H}\left(A_{t} / \dot{Q}_{L}\right)_{\min }\right]$.

It was found that Eq. (13) is applicable for minimum total area also. Above analytical models can be further improved by considering component pressure drop and process parameter dependent irreversibility parameter.

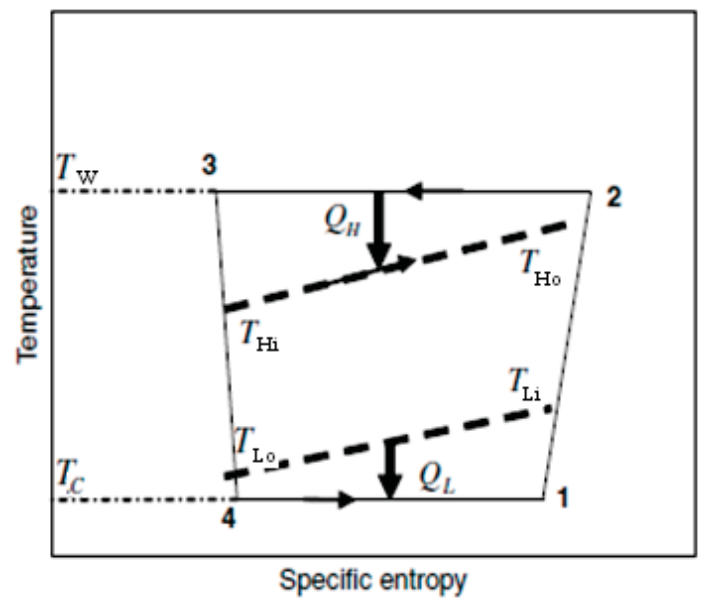

Figure 2: Irreversible refrigerator with finite heat reservoirs.

\section{Analytical Optimization with Finite Heat Reservoirs 3.1 Problem Formulation}

Practically, the heat reservoirs have finite heat capacities. Consequently, the temperature in the reservoirs is not constant. Therefore, the system performance depends on the magnitude of the heat capacity and the temperature variations of the reservoirs. This problem formulation is based on the cycle $\mathrm{T}-\mathrm{s}$ diagram of Figure 2 in which the heat source supplies the refrigeration load and the heat sink receives the heat rejection load in both counterflow heat exchangers. This refrigeration cycle is composed of the following processes: (i) polytropic irreversible compression, (ii) isothermal heat rejection, (iii) polytropic irreversible expansion and (iv) isothermal heat addition. Earlier stated assumptions can also apply in this case except (i). Here, reservoirs have finite heat capacity leading to variable temperature (i.e. single phase heat transfer for heat reservoir fluids). Eq. (1) is also applicable for irreversibility factor. For the present case, the effectiveness of both-side counterflow heat exchangers are given by [18]:

$$
\begin{aligned}
& \varepsilon_{L}=1-\exp \left(-U_{L} A_{L} / C_{L}\right) \\
& \varepsilon_{H}=1-\exp \left(-U_{H} A_{H} / C_{H}\right)
\end{aligned}
$$

\subsection{Optimization Results}

Cooling capacity of the irreversible cycle for given effectiveness of cold and hot side heat exchangers can be expressed by [18]:
$\dot{Q}_{L}=\left[\frac{T_{W} / T_{C}}{\varepsilon_{H} C_{H}\left(T_{W}-T_{H i}\right)}+\frac{1}{\varepsilon_{L} C_{L}\left(T_{L i}-T_{C}\right)}\right]^{-1}$

Combining with Eq. (5), warm and cold working fluid temperatures can be optimized to get maximum cooling load. Chiou et al. [18] have optimized for endoreversible cycle only, which is re-derived in this study for irreversible cycle. For given COP, the optimum working fluid temperatures can be derived as:

$$
T_{C, \text { opt }}=\left(\phi X T_{H i}+\sqrt{\frac{\varepsilon_{L} C_{L}}{\varepsilon_{H} C_{H}}} T_{L i}\right) /\left(1+\sqrt{\frac{\varepsilon_{L} C_{L}}{\varepsilon_{H} C_{H}}}\right)
$$

$$
T_{W, o p t}=\left(T_{H i}+\sqrt{\frac{\varepsilon_{L} C_{L}}{\varepsilon_{H} C_{H}}} \frac{T_{L i}}{\phi X}\right) /\left(1+\sqrt{\frac{\varepsilon_{L} C_{L}}{\varepsilon_{H} C_{H}}}\right)
$$

Corresponding maximum cooling load is given by,

$$
\dot{Q}_{L, \text { max }}=\left[\varepsilon_{L} C_{L} \varepsilon_{H} C_{H}\left(\sqrt{\varepsilon_{H} C_{H}}+\sqrt{\varepsilon_{L} C_{L}}\right)^{-2}\right]\left(T_{L i}-\phi X T_{H i}\right)
$$

El-Din [32] obtained optimum area inventory of hot and cold side heat exchanger to get maximum cooling capacity based on stated assumptions. He derived the following equation for cooling capacity for given COP:

$Q_{L}=\frac{(1-\alpha) \alpha A_{t} E_{H} T_{H i}\left[\left(T_{L i} / T_{H i}\right)-\left(T_{C} / T_{W}\right)\right]}{(1-\alpha) E_{H} / E_{L}+\phi \alpha}$

where, $E_{L}=\varepsilon_{L} C_{L} / A_{L}$ and $E_{H}=\varepsilon_{H} C_{H} / A_{H}$.

For total area as constraint, the following equation has been derived for optimum area ratio to get maximum cooling capacity [32]:

$\alpha_{\text {opt }}=\sqrt{\phi} / \sqrt{E_{H} / E_{L}}$

And hence the corresponding maximum cooling capacity given by:

$Q_{L, \max }=\frac{A_{t} E_{H} T_{H i}\left[\left(T_{L i} / T_{H i}\right)-\left(T_{C} / T_{W}\right)\right]}{\left(\sqrt{E_{H} / E_{L}}+\sqrt{\phi}\right)^{2}}$

Above derivation has been done for given COP and he found that the effect of irreversibility parameter diminished for $E_{h} / E_{c}>1$. It may be noted that the above derivation is improper and full of errors as $E_{L}$ and $E_{H}$ are not constant. Hence, the above optimum correlations can't yield good prediction. Li et al. [56] have derived optimum allocation of heat exchanger area for the heat pump with variable temperature heat reservoirs by considering heat leaks.

$\mathrm{Yu}$ et al. [43] maximized the COP of irreversible Carnot-like refrigerator for given finite mass flow rate. They defined relative mass flow rates by, $R_{L}=\varepsilon_{L} m_{L} / m_{r}$ and $R_{H}=\varepsilon_{H} m_{H} / m_{r}$, and total by, $R=R_{L}+R_{H}$. They found the following expression for optimum relative mass flow rate allocation ratio $\left(x=R_{L} / R\right)$, 


$$
x_{\text {opt }}=\frac{1-\sqrt{1-\left(1-\phi c_{p L} / c_{p H}\right)\left(1-\phi\left(s_{1}-s_{4}\right) / R c_{p H}\right)}}{1-\phi c_{p L} / c_{p H}}
$$

However, mass flow rate should be same for steadystate. Sarkar and Bhattacharyya [37] analytically minimized the overall heat transfer area per unit capacity of refrigeration system considering both internal and external irreversibilities with variable temperature (finite capacity) heat reservoirs. Hot and cold side refrigerant temperatures and heat transfer area ratios were optimized to attain this goal. Using Eqs. (24-25), the total heat exchanger area required for unit cooling output is given by,

$$
A_{\tau}=\frac{C_{H}}{U_{H}} \ln \left[1-\frac{\phi T_{W} / T_{C}}{C_{H}\left(T_{W}-T_{H i}\right)}\right]^{-1}+\frac{C_{L}}{U_{L}} \ln \left[1-\frac{1}{C_{L}\left(T_{L i}-T_{C}\right)}\right]^{-1}
$$

After replacing $\mathrm{T}_{\mathrm{W}}$ by Eq. (5) in the above equation and equating the derivative of $A_{t}$ with respect to $T_{C}$ to zero, the following expressions for the optimal cold and hot side working fluid temperatures can be obtained:

$$
\begin{aligned}
& T_{C, \text { opt }}=\left[G-\sqrt{G^{2}-4(1-\delta / \phi) J}\right] /[2(1-\delta / \phi)] \\
& T_{W, \text { opt }}=\left[G-\sqrt{G^{2}-4(1-\delta / \phi) J}\right] /[2(1-\delta / \phi) \phi X]
\end{aligned}
$$

where,

$$
\begin{aligned}
& G=2\left(T_{L i}-\delta X T_{H i}\right)-\left(1 / C_{L}+\delta / C_{H}\right) \\
& J=T_{L i}^{2}-\delta \phi\left(X T_{H i}\right)^{2}-T_{L i} / C_{L}-\delta \phi X T_{H i} / C_{H}
\end{aligned}
$$

Substituting the optimal working fluid temperatures, Eq. (34) will yield the minimum overall heat transfer area per unit cooling load. Above optimum equations were wellverified with numerical results of ammonia refrigeration cycle.

\section{Numerical Verifications}

\subsection{Mathematical Modeling and Simulation}

In this study, the analytical optimization results of irreversible refrigeration cycle have been reviewed and verified by comparing with the results obtained from an elaborate numerical simulation of transcritical $\mathrm{CO}_{2}$ refrigeration cycle. The actual $\mathrm{CO}_{2}$ based refrigerator with constant or variable temperature heat reservoirs is shown in Figure 3, which consists of evaporator, compressor, gas cooler and expansion device. As shown, the heat rejection (gas cooling) from $\mathrm{CO}_{2}$ occurs at variable temperature as it operated in supercritical zone [57-58]. Whereas, majority of heat rejection for conventional refrigerant based cycle occurs at constant temperature (condensation), which is very similar to irreversible Carnot-like cycle. This is the major difference between $\mathrm{CO}_{2}$ cycle and the irreversible Carnot-like cycle. Both the heat exchangers (evaporator and gas cooler) are of counter flow type. The cycle has been modeled based on first law of thermodynamics (energy balance). The following assumptions have been made for the present analysis:

(i) Cycle is operating at steady state condition.

(ii) Evaporator outlet (state 1) is saturated vapor.

(iii) Both compression and expansion processes are irreversible adiabatic (iv) Both evaporation and gas cooling are isobaric

(v) Expansion process is isenthalpic

(vi) Change in kinetic and potential energies are neglected for all components

(vii) Compressor has fixed isentropic efficiency of $80 \%$

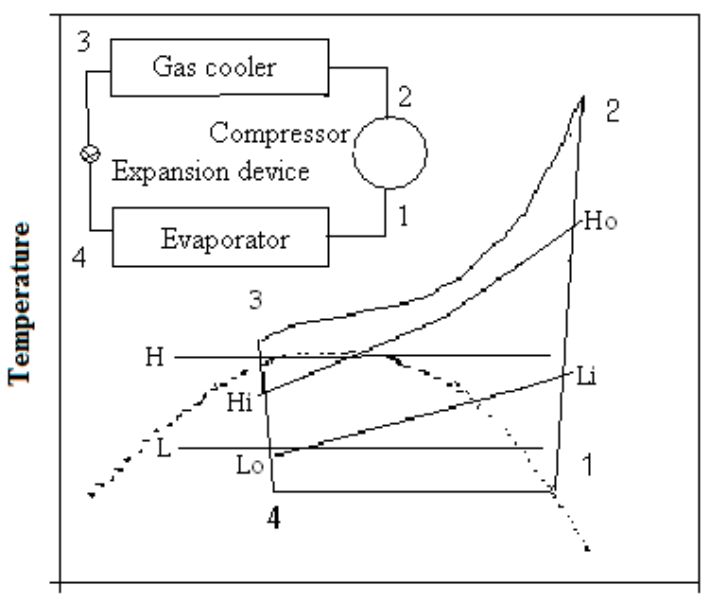

Entropy

Figure 3: Transcritical $\mathrm{CO}_{2}$ refrigeration cycle.

Cold side refrigerant temperature $\left(T_{C}\right)$ is same as the evaporator temperature $\left(T_{1}\right)$. However, unlike to subcritical cycle, the heat rejection process for refrigerant in transcritical $\mathrm{CO}_{2}$ cycle is not isothermal. Hence, to make $\mathrm{CO}_{2}$ refrigeration cycle equivalent to reversed Carnot cycle, the hot side refrigerant temperature $\left(T_{W}\right)$ has been approximated as the thermodynamic average temperature of refrigerant in the gas cooler. This is defined as the ratio of enthalpy change during the heat rejection process to the entropy change thereof [33]:

$T_{W}=\left(h_{2}-h_{3}\right) /\left(s_{2}-s_{3}\right)$

The irreversibility parameter has been evaluated by,

$\phi=\left(s_{2}-s_{3}\right) /\left(s_{1}-s_{4}\right)$

As discussed earlier, the irreversibility parameter is dependent on fluid operating parameters. Hence, it was calculated based on simulated data for numerical verification. The cooling and heating capacities for infinite reservoirs can be written as:

$\dot{Q}_{L}=A_{L} U_{L}\left(T_{L}-T_{C}\right)=m_{r}\left(h_{1}-h_{4}\right)$

$\dot{Q}_{H}=A_{H} U_{H}\left(T_{2}-T_{3}\right) / \ln \left[\left(T_{2}-T_{H}\right) /\left(T_{3}-T_{H}\right)\right]=m_{r}\left(h_{2}-h_{3}\right)$

Similarly for finite capacity reservoirs:

$$
\begin{aligned}
\dot{Q}_{L} & =A_{L} U_{L}\left(T_{L i}-T_{L o}\right) / \ln \left[\left(T_{L i}-T_{1}\right) /\left(T_{L o}-T_{1}\right)\right] \\
& =m_{r}\left(h_{1}-h_{4}\right)=C_{L}\left(T_{L i}-T_{L o}\right) \\
\dot{Q}_{H} & =A_{H} U_{H}\left[\left(T_{2}-T_{H o}\right)-\left(T_{3}-T_{H i}\right)\right] / \ln \left[\left(T_{2}-T_{H o}\right) /\left(T_{3}-T_{H i}\right)\right] \\
& =m_{r}\left(h_{2}-h_{3}\right)=C_{H}\left(T_{H o}-T_{H i}\right)
\end{aligned}
$$

The COP of the cycle is given by, 
$C O P=\dot{Q}_{L} /\left(\dot{Q}_{H}-\dot{Q}_{L}\right)=\left(h_{1}-h_{4}\right) /\left(h_{2}-h_{1}\right)$

To evaluate the variation of cooling load $\left(\dot{Q}_{L}\right)$ with heat transfer surface area ratio $\left(A_{H} / A_{L}\right)$ and the variation of total heat transfer surface area $\left(\mathrm{A}_{\mathrm{L}}+\mathrm{A}_{\mathrm{H}}\right)$ with refrigerant temperature for certain value of COP, above equation have been effectively used. However, to evaluate the variation of cooling load with refrigerant temperature, the heat transfer parts of above equations have been modified as follows:

The cold side heat transfer for given residence time is,

$Q_{L} \begin{cases}=A_{L} U_{L}\left(T_{L}-T_{1}\right) t_{C} & \text { (infinite) } \\ =\frac{A_{L} U_{L}\left(T_{L i}-T_{L o}\right) t_{C}}{\ln \left[\left(T_{L i}-T_{1}\right) /\left(T_{L o}-T_{1}\right)\right]} & \text { (finite) }\end{cases}$

Hence, the cold side heat transfer rate is given by,

$\dot{Q}_{L} \begin{cases}=\left(t_{C} / t\right) A_{L} U_{L}\left(T_{L}-T_{1}\right) & \text { (infinite) } \\ =\frac{\left(t_{C} / t\right) A_{L} U_{L}\left(T_{L i}-T_{L o}\right)}{\ln \left[\left(T_{L i}-T_{1}\right) /\left(T_{L o}-T_{1}\right)\right]} & \text { (finite) }\end{cases}$

Similarly, the hot side heat transfer rate is given by,

$$
\dot{Q}_{H} \begin{cases}=\frac{\left(t_{W} / t\right) A_{H} U_{H}\left(T_{2}-T_{3}\right)}{\ln \left[\left(T_{2}-T_{H}\right) /\left(T_{3}-T_{H}\right)\right]} & \text { (infinite) } \\ =\frac{\left(t_{W} / t\right) A_{H} U_{H}\left[\left(T_{2}-T_{H o}\right)-\left(T_{3}-T_{H i}\right)\right]}{\ln \left[\left(T_{2}-T_{H o}\right) /\left(T_{3}-T_{H i}\right)\right]} & \text { (finite) }\end{cases}
$$

Where, the total residence time, $t=t_{C}+t_{W}$ (neglecting expansion and compression times). The simulation code has been developed incorporating the earlier developed $\mathrm{CO}_{2}$ property subroutine [59]. Effective iteration techniques have been used as applicable.

\subsection{Results and Discussion}

The following fixed values have been taken in simulation: $U_{L}=U_{H}=0.5 \mathrm{~kW} / \mathrm{m}^{2} \mathrm{~K}$, gas cooler pressure = 100bar, COP $=2.5, T_{L}=285 \mathrm{~K}, T_{H}=310 \mathrm{~K}, T_{L i}=305 \mathrm{~K}$, $T_{H i}=310 \mathrm{~K}, C_{L}=C_{H}=0.05 \mathrm{~kW} / \mathrm{K}$.

The variations of cooling capacity with $T_{C}$ for $A_{L}=$ $A_{H}=0.25 \mathrm{~m}^{2}$ are shown in Figures 4 and 5 for constant and variable temperature reservoirs respectively. As shown, the numerical values of optimum cold and warm side refrigerant temperatures differ by not more than $1 \%$, the corresponding maximum cooling capacity differs significantly by $13.5 \%$ and the optimum residence time ratio differs by about $4.8 \%$ with the analytical results for the constant temperature reservoirs. On the other hand, the numerical values of optimum cold and warm side refrigerant temperatures differ by about $2.1 \%$ and the corresponding maximum cooling capacity differs significantly by $22 \%$ with the analytical results for the variable temperature reservoirs.

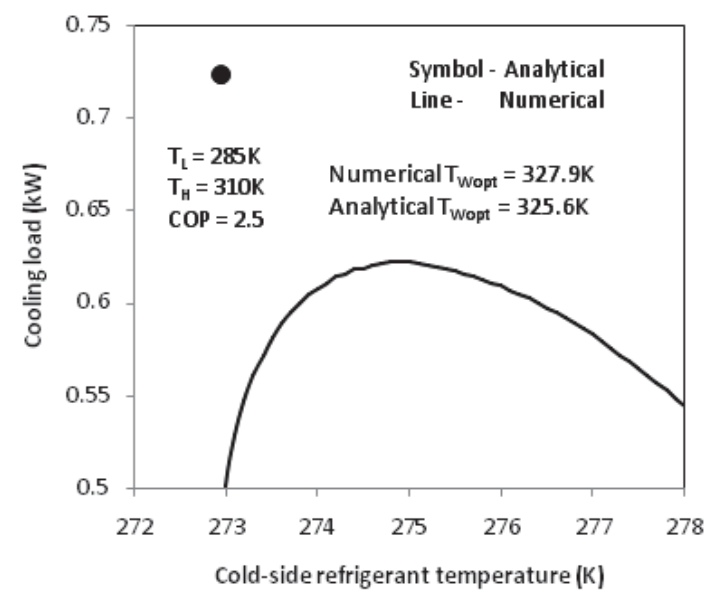

Figure 4: Cooling load v/s refrigerant temperature for infinite capacity reservoirs.

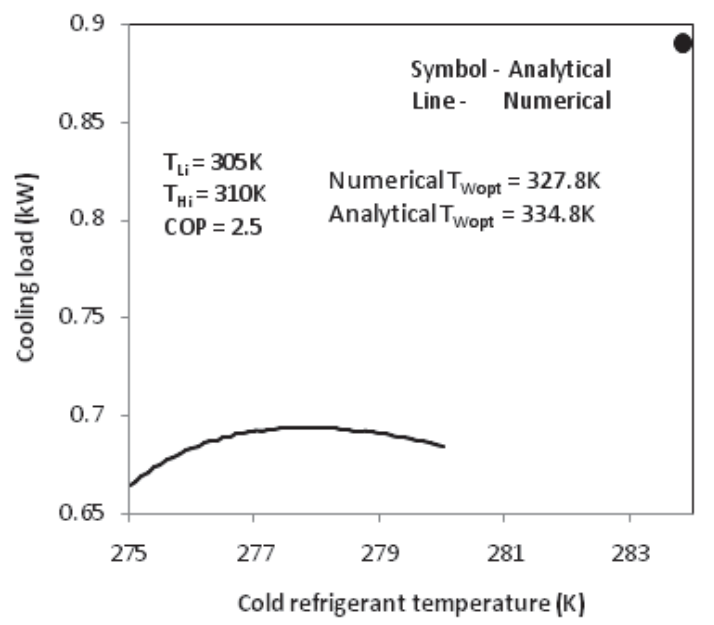

Figure 5: Cooling load v/s refrigerant temperature for finite capacity reservoirs.

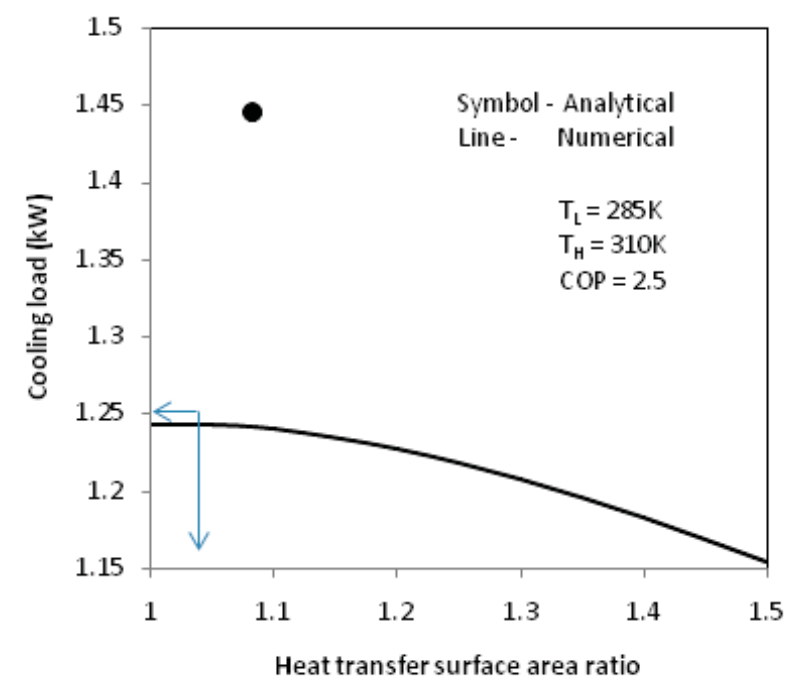

Figure 6: Cooling load v/s area ratio for infinite capacity reservoirs. 


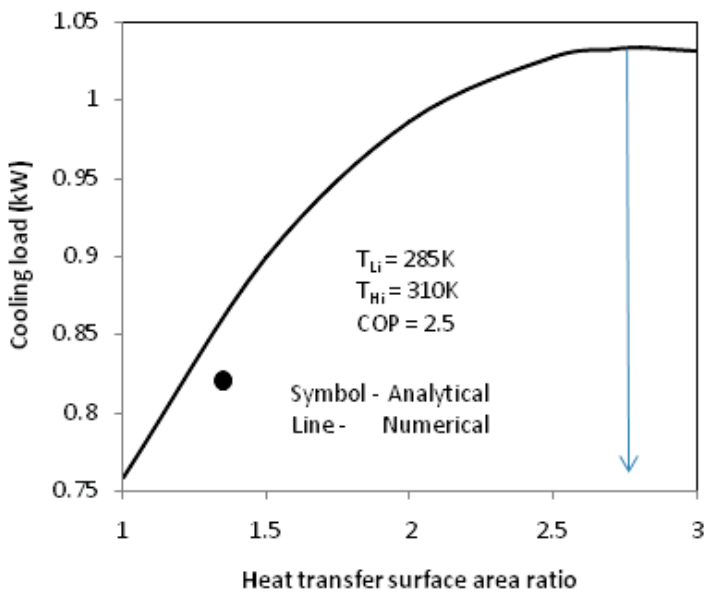

Figure 7: Cooling load v/s area ratio for finite capacity reservoirs.

The variations of cooling capacity with heat transfer area ratio for total area of $0.5 \mathrm{~m}^{2}$ are shown in Figures 6 and 7 for constant and variable temperature reservoirs respectively. For given area allocation, cooling load has been calculated using Eqs. (37-43) applying suitable iteration technique. As shown, the numerical values of optimum heat transfer area ratio differs by about $4.9 \%$ and the corresponding maximum cooling capacity differs significantly by $14 \%$ with the analytical results for the constant temperature reservoirs. These deviations are more compared to the deviation with subcritical refrigeration cycle [34]. On the other hand, the numerical values of optimum heat transfer area ratio differs very significantly by about $100 \%$ and the corresponding maximum cooling capacity also differs significantly by $26 \%$ with the analytical results for the variable temperature reservoirs. Furthermore, the present results have also compared with the optimization results of simulated transcritical $\mathrm{CO}_{2}$ heat pump for simultaneous water cooling and heating considering real system components, heat transfer and fluid flow effects [59]. Comparison showed that that the simulated optimum heat transfer area ratio is differ by about $72 \%$ with the predicted value based on other fixed data of simulated system. Above discussion reveals that either the finite time thermodynamics model is not suitable for this optimization or this may happen due to improper derivation of optimum area ratio (Eq. 31 is not explicit).

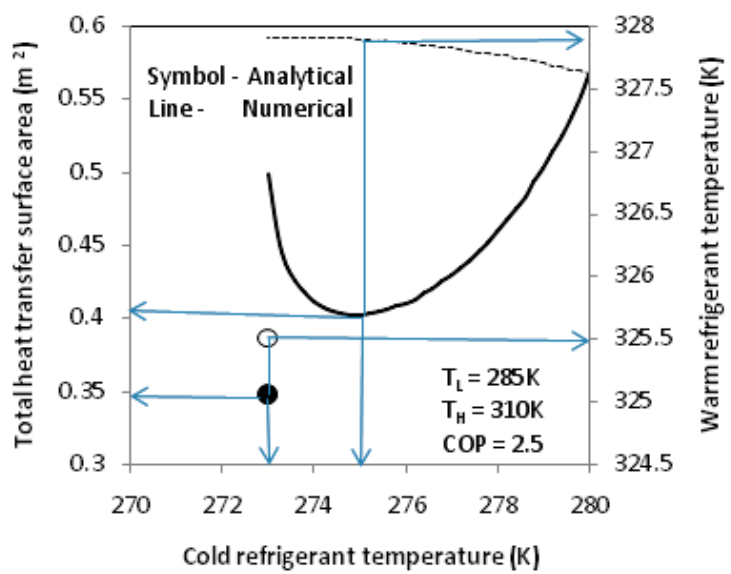

Figure 8: Overall heat transfer area $\mathrm{v} / \mathrm{s}$ refrigerant temperature for infinite capacity reservoirs.
The variations of total heat transfer surface area per unit cooling capacity $(1 \mathrm{~kW})$ with $T_{C}$ are shown in Figures 8 and 9 for constant and variable temperature reservoirs respectively. As shown, the numerical values of optimum cold and warm side refrigerant temperatures differ by not more than $0.75 \%$ and the corresponding minimum heat transfer area differs by about $15.7 \%$ with the analytical results for the constant temperature reservoirs. On the other hand, the numerical values of optimum cold and warm side refrigerant temperatures differ by within $2.9 \%$ and the corresponding minimum heat transfer area differs by about $7.4 \%$ with the analytical results for the variable temperature reservoirs. These deviations are also more compared to the deviation with subcritical refrigeration cycle [36-37]. Present discussion shows that the deviations of analytical results with the numerical results are reasonable in most of the cases. Model may be improved by taking isobaric heat rejection.

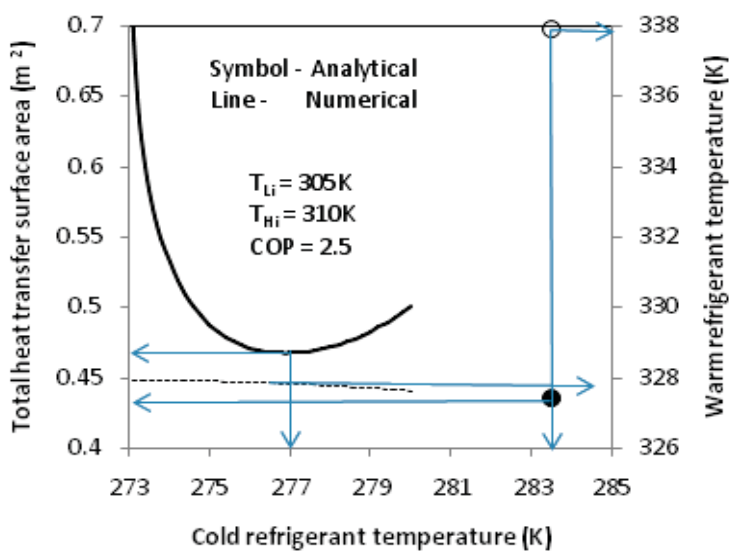

Figure 9: Overall heat transfer area $v / s$ refrigerant temperature for finite capacity reservoirs.

\section{Conclusions}

In the present study, analytical optimizations of working fluid temperatures, ratio of residence times for heat addition and heat rejection, and heat transfer surface area ratio of irreversible Carnot-like refrigerator based on maximization of cooling load or cooling COP and minimization of overall heat transfer surface area have been reviewed for both constant temperature and variable temperature heat reservoirs. Analytical results have been verified with the numerical optimization results of transcritical $\mathrm{CO}_{2}$ refrigeration cycle as well. Comparison shows that the analytical results deviate reasonably with the numerical results in most of the cases, although the deviations are more compared to the subcritical cycles. Present review and verification reveal that optimization results of irreversible Carnot-like refrigerator are applicable for transcritical refrigeration cycle also. However, better prediction can be achieved by incorporating process parameters dependent $\phi$ in optimization.

\section{Nomenclature}

A heat transfer surface area

$\mathrm{A}_{t} \quad$ total surface area of the heat exchangers

C heat capacity of reservoir fluid

$\mathrm{C}_{\mathrm{p}} \quad$ specific isobaric heat capacity

$\mathrm{h} \quad$ specific enthalpy

$\dot{Q} \quad$ heat transfer rate

m mass flow rate 


$\begin{array}{ll}\mathrm{S} & \text { entropy } \\ \mathrm{T} & \text { temperature } \\ \mathrm{t} & \text { residence time } \\ \mathrm{U} & \text { overall heat transfer coefficient } \\ \alpha & \text { heat transfer area ratio } \\ \delta & \text { overall heat transfer coefficient ratio } \\ \varepsilon & \text { heat exchanger effectiveness } \\ \varnothing & \text { irreversibility parameter } \\ \text { Subscripts } \\ \mathrm{C} & \text { cold side working fluid } \\ \mathrm{H} & \text { high temperature reservoir/heat exchanger } \\ \mathrm{i} & \text { reservoir inlet } \\ \mathrm{L} & \text { low temperature reservoir/heat exchanger } \\ \text { max } & \text { maximum } \\ \text { min } & \text { minimum } \\ \text { o } & \text { reservoir outlet } \\ \text { opt } & \text { optimum } \\ \mathrm{r} & \text { refrigerant (working fluid) } \\ \mathrm{W} & \text { hot side working fluid }\end{array}$

\section{References:}

[1] Y. Wang, M. Li, Z. C. Tu, A. C. Hernández, J. M. M. Roco, Coefficient of performance at maximum figure of merit and its bounds for low-dissipation Carnotlike refrigerators, Physical Review E - Statistical, Nonlinear, Soft Matter Physics, 86, 011127, 2012.

[2] A. Bejan, Entropy generation minimization: the new thermodynamics of finite-size devices and finite-time processes, J. Appl. Physics, 79, 1191-1218, 1996.

[3] L. Chen, C. Wu, F. Sun, Finite time thermodynamic optimization or entropy generation minimization of energy systems, J. Non-Equil. Therm., 24, 327-359, 1999.

[4] A. Durmayaz, O. S. Sogut, B. Sahin, H. Yavuz, Optimization of thermal system based on finite-time thermodynamics and thermoeconomics, Prog. Energy Comb. Sci., 30, 175-217, 2004.

[5] B. Andresen, Current Trends in Finite-Time Thermodynamics, Angew. Chem. Int. Ed., 50, 26902705, 2011.

[6] H. F. Leff, W. D. Teeters, EER, COP, and 2nd law efficiency for air-conditioners, American J. Physics, 46, 19-22, 1978.

[7] C. H. Blanchard, Coefficient of performance for finite speed heat pump, J. Applied Physics, 51, 2471-2472, 1980.

[8] A. Bejan, Theory of heat transfer-irreversible refrigeration plants, Int. J. Heat Mass Transfer, 32, 1631-1639, 1989.

[9] Z. Yan, J. Chen, A class of irreversible Carnot refrigeration cycles with a general heat transfer law, $J$. of Physics D: Applied. Physics, 23, 136-141, 1990.

[10] D. C. Agrawal, V. J. Menon, Performance of a Carnot refrigerator at maximum cooling power, J. of Physics A: Mathematical \& General, 23, 5319-5326, 1990.

[11] S.A. Klein, Design considerations for refrigerating cycles, Int. J. Refrigeration, 15, 181-185, 1992.
[12] A. Bejan, Power and refrigeration plants for minimum heat exchanger inventory, J. Energy Res. Tech., 115, 148-150, 1993.

[13] G. Grazzini, Irreversible refrigerators with isothermal heat exchanges, Int. J. Refrigeration, 16, 101-106, 1993.

[14] J. Chen, New performance bounds of a class of irreversible refrigerators, Journal Physics A: Math. Gen. 27, 6395-6401, 1994.

[15] C. Wu, Maximum obtainable specific cooling load of a refrigerator, Energy Conversion Mgmt., 36, 7-10, 1995.

[16] J. Chen, C. Wu, Design considerations of primary performance parameters for irreversible refrigeration cycles, Int. J. Amb. Energy, 16, 17-22, 1995.

[17] W. Z. Chen, F. R. Sun, S. M. Cheng, L. G. Chen, Study on optimal performance and working temperatures of endoreversible forward and reverse Carnot cycles, Int. J. Energy Res., 19, 751-759, 1995.

[18] J. S. Chiou, C. J. Liu, C. K. Chen, The performance of an irreversible carnot refrigeration cycle, J. Physics D-Applied Physics, 28, 1314-1318, 1995.

[19] C. Y. Cheng, C. K. Chen, Performance optimization of an irreversible heat pump, J. Physics. D: Appl. Physics, 28, 2451-2454, 1995.

[20] M. A. Ait-Ali, The maximum coefficient of performance of internally irreversible refrigerators and heat pumps, J. Physics D-Applied Physics, 29, 975-980, 1996.

[21] L. Chen, C. Wu, F. Sun, Heat transfer effect on the specific cooling load of refrigerators, Applied Thermal Engg., 16, 989-997, 1996.

[22] C. Wu, Performance of an endoreversible Carnot refrigerator, Energy Conversion Mgmt., 37, 15091512, 1996.

[23] C. Wu, L. Chen, F. Sun, W. Chen, General performance characteristics of a finite-speed Carnot refrigerator, Applied Thermal Eng., 16, 299-303, 1996.

[24] L. Chen, C. Wu, F. Sun, Heat transfer effect on the specific heating load of heat pumps, Appl. Thermal Eng., 17, 103-110, 1997.

[25] L. Chen, C. Wu, F. Sun, Heat pump performance with internal heat leak, Int. J. Ambient Energy, 18, 129134, 1997.

[26] L. Chen, C. Wu, F. Sun, A generalized model of a real refrigerator and its performance, Applied Thermal Eng., 17, 401-412, 1997.

[27] F. Sun, W. Chen, L. Chen, C. Wu, Optimal performance of an endoreversible Carnot heat pump, Energy Conversion Mgmt., 38, 1439-1443, 1997.

[28] L. Chen, C. Wu, F. Sun, Influence of internal heat leak on the performance of refrigerators, Energy Conversion Mgmt., 39, 45-50, 1998. 
[29] L. Chen, F. Sun, N. Ni, C. Wu, Optimal configuration of a class of two heat reservoir refrigeration cycles, Energy Conversion Mgmt., 39, 767-773, 1998.

[30] C. Wu, L. Chen, F. Sun, Optimization of steady flow heat pumps, Energy Conversion Mgmt., 39, 445-453, 1998.

[31] M. M. S. El-Din, Optimization of totally irreversible refrigerators and heat pumps, Energy Conversion Mgmt., 40, 423-36, 1999.

[32] M. M. S. El-Din, Performance analysis of heat pumps and refrigerators with variable reservoir temperatures, Energy Convers. Mgmt., 42, 201-216, 2001.

[33] L. Chen, F. Sun, C. Wu, Effect of heat transfer law on the performance of a generalized irreversible Carnot refrigerator, Journal Non-Equilibrium Thermo., 26, 291-304, 2001.

[34] L. Chen, F. Sun, C. Wu, Optimal allocation of heat exchanger area for refrigeration and air-conditioning plants, Appl. Energy 77, 339-354, 2004.

[35] J. Sarkar, S. Bhattacharyya, M. Ramgopal, Thermodynamic optimization of irreversible heat pumps. Archives Thermo., 29, 59-68, 2008.

[36] J. Sarkar, S. Bhattacharyya, M. Ramgopal, Analytical minimization of overall conductance and heat transfer area in refrigeration and heat pump systems and its numerical confirmation, Energy Conversion Mgmt., 48, 1245-1250, 2007.

[37] J. Sarkar, S. Bhattacharyya, Overall conductance and heat transfer area minimization of refrigerators and heat pumps with finite heat reservoirs, Energy Conversion Mgmt., 48, 803-808, 2007.

[38] J. Li, L. Chen, F. Sun, Cooling load and coefficient of performance optimization for a generalized irreversible Carnot refrigerator with heat transfer law $\mathrm{q} \propto\left(\Delta \mathrm{T}^{\mathrm{n}}\right)^{\mathrm{m}}$, IMechE-E J. Process Mech. Eng., 222, 5562, 2008.

[39] C. Petre, M. Feidt, M. Costea, S. Petrescu, A model for study and optimization of real-operating refrigeration machines, Int. J. Energy Research, 33, 173-179, 2009.

[40] S. K. Tyagi, Q. Wang, P. Xia, G. M. Chen, Optimisation of an irreversible Carnot refrigerator working between two reservoirs, Int. J. Exergy, 7, 7688, 2010.

[41] J. Li, L. Chen, F. Sun, Fundamental optimal relation of a generalized irreversible Carnot heat pump with complex heat transfer law, Pramana-J. Physics, 74, 219-230, 2010.

[42] M. Feidt, Thermodynamics applied to reverse cycle machines, a review, Int. J. Refrigeration, 33, 13271342, 2010.

[43] J. Yu, Y. Zhou, Y. Liu, Performance optimization of an irreversible Carnot refrigerator with finite mass flow rate, Int. J. Refrigeration, 34, 567-572, 2011.

[44] N. E. Wijeysundera, An irreversible-thermodynamic model for solar-powered absorption cooling systems, Solar Energy, 68, 69-75, 2000.
[45] Y. Ust, B. Sahin, Performance optimization of irreversible refrigerators based on a new thermoecological criterion, Int. J. Refrigeration, 30, 527-534, 2007.

[46] Y. Ust, A. V. Akkaya, A. Safa, Analysis of a vapour compression refrigeration system via exergetic performance coefficient criterion, J. Energy Institute, 84, 66-72, 2011.

[47] L. Chen, J. Li, F. Sun, Ecological optimization of a generalized irreversible Carnot refrigerator in the case of $\mathrm{Q}^{\alpha}\left(\Delta \mathrm{T}^{\mathrm{n}}\right)^{\mathrm{m}}$, Int. J. Sust. Energy, 31, 59-72, 2012.

[48] H. Wang, G. Wu, Thermosize effects and irreversibility on the performance of a macro/nano scaled refrigeration cycle, J. Applied Physics, 112, Article number 084325, 2012.

[49] Y. Zhou, J. Yu, Generalized model of internally irreversible carnot refrigerators with finite mass flow rate, J. Therm. heat transfer, 27, 364-367, 2013.

[50] P. A. N. Wouagfack, R. Tchinda, Finite-time thermodynamics optimization of absorption refrigeration systems: A review, Renewable Sustainable Energy Reviews, 21, 524-536, 2013.

[51] M. Yari, Exergetic analysis of the vapour compression refrigeration cycle using ejector as an expander, Int. Journal Exergy, 5, 326-340, 2008.

[52] M. Yari, Second law optimization of two-stage transcritical $\mathrm{CO}_{2}$ refrigeration cycles in the cooling mode operation, IMechE Pt A-Journal Power Energy, 223, 551-561, 2009.

[53] P. K. Bhardwaj, S. C. Kaushik, S. Jain, Finite time thermodynamic optimization of irreversible Rankine cycle heat pump system, Indian J. Pure \& Applied Physics, 41, 515-521, 2003.

[54] M. E. H. Assad, Cooling load optimization of an irreversible refrigerator with combined heat transfer, Int. Journal Energy Env., 4, 377-386, 2013.

[55] B. A. Qureshi, S. M. Zubair, Cost optimization of heat exchanger inventory for mechanical subcooling refrigeration cycles, Int. J. Refrigeration, 36, 12431253, 2013.

[56] X. Li, Z. Yang, X. Shi, Y. Shi, Q. Zhang, Optimal allocation of heat exchanger area for vaporcompression heat pump plants, Advanced Materials Research, 433-440, 817-822, 2012.

[57] K. Srinivasan, Y. K. Lim, J. C. Ho, N. E. Wijeysundera, Exergetic analysis of carbon dioxide vapour compression refrigeration cycle using the new fundamental equation of state, Energy Conversion Mgmt, 44, 3267-3278, 2003.

[58] J. Sarkar, Review on cycle modifications of transcritical $\mathrm{CO}_{2}$ refrigeration and heat pump systems, J. Adv. Research Mechanical Eng., 1, 22-29, 2010.

[59] J. Sarkar, S. Bhattacharyya, M. Ramgopal, Simulation of a transcritical $\mathrm{CO}_{2}$ heat pump cycle for simultaneous cooling and heating applications, Int. J. Refrigeration, 29, 735-743, 2006. 